\title{
Experimental study of the reduction of field emission by gas injection in vacuum for accelerator applications
}

\author{
K. Almaksour, ${ }^{1,2}$ M. J. Kirkpatrick, ${ }^{2,}$ Ph. Dessante, ${ }^{2}$ E. Odic, ${ }^{2}$ A. Simonin, ${ }^{3}$ \\ H. P. L. de Esch, ${ }^{3}$ B. Lepetit, ${ }^{4}$ D. Alamarguy, ${ }^{1}$ F. Bayle, ${ }^{5}$ and Ph. Teste ${ }^{1}$ \\ ${ }^{1}$ LGEP-CNRS/Université Paris 6 \& 11/SUPELEC, Gif-sur-Yvette, France \\ ${ }^{2}$ SUPELEC-DESE, Gif-sur-Yvette, France \\ ${ }^{3}$ CEA, IRFM, F-13108 Saint-Paul-lez-Durance, France \\ ${ }^{4}$ LCAR, IRSAMC, Université de Toulouse, Toulouse, France \\ ${ }^{5}$ IEF-Université Paris-Sud, Orsay, France \\ (Received 12 June 2014; published 3 October 2014)
}

\begin{abstract}
Field emission current from surfaces under vacuum and at high field strengths can be reduced by the injection of gas into the evacuated volume. In this paper, the effects of $\mathrm{H}_{2}, \mathrm{He}, \mathrm{N}_{2}$, and $\mathrm{Ar}$ on this "dark" current emitted from a tungsten carbide point cathode for $2 \mathrm{~cm}$ gap distance is studied. Exposure to any of these gases at pressures on the order of $10^{-3}-10^{-2} \mathrm{~Pa}$ was found to reduce the emission current by up to $90 \%$ with a time constant on the order of $\sim 1$ minute as compared to the current at $10^{-6} \mathrm{~Pa}$. The effect was strongly dependent on the gas nature, with Ar and $\mathrm{N}_{2}$ having larger effects at lower pressures than $\mathrm{He}$ and $\mathrm{H}_{2}$. The reduction was reversible, with the current increasing to near its original value with a time constant on the order of $\sim 1-10$ minutes after pumping down. The effect of the gas remained in the absence of electric field, whatever the gas pressure. Mechanisms for these and related phenomena are discussed.
\end{abstract}

PACS numbers: 51.50.+v, 07.30.-t, 52.50.Gj, 52.80.Vp

\section{INTRODUCTION}

Under high electric field and in vacuum, electronic emission from sites on a cathode lead to a low intensity current, the field emission current or so-called "dark" current [1]. While field emission is desirable in many circumstances it is nonetheless a problem in many others, notably for the neutral beam injector systems for the planned fusion power plants (ITER and DEMO) [2-4].

Field emission current poses two distinct problems for the injector systems. First, in the ITER $1 \mathrm{MV}$ injector system, for example, it will lead to a voltage limitation for a given size power supply, and if it exceeds certain limits, will in principle necessitate a scale-up. The other problem is that dark current is known to be associated with breakdown in vacuum [5], posing challenges to long-term, maintenance-free injector operation.

One well-known method of reducing the intensity of field emission current is by the injection of gas, typically raising the pressure of the enclosure from high or ultrahigh vacuum to pressures on the order of $10^{-3}-10^{-2} \mathrm{~Pa}$ [6-9]. While this effect has been known for quite some time, some disagreement still persists in the literature over the exact mechanism leading to it. Many, following the work of

\section{"mike.kirkpatrick@gmail.com}

Published by the American Physical Society under the terms of the Creative Commons Attribution 3.0 License. Further distribution of this work must maintain attribution to the author(s) and the published article's title, journal citation, and DOI.
Alpert [6], consider ion bombardment of microscale or nanoscale emitters to be responsible [5,8,9]. Another possibility which has been suggested is that ions are responsible for the effect not by bombarding and sputtering of emitter structures but by implantation within dielectric inclusions [10]. It has also been proposed that, as modification of gas adsorption can be caused by the high electric field at the emitter tips [11], that this or other perturbations of the balance between sticking and ejection processes for adsorbates (or perhaps other contaminants such as vacuum pump lubricants) at the surface may change the work function $[12,13]$ and thus be responsible for the effect.

The present paper seeks a better understanding of this effect and involves the experimental investigation of several issues: the nature of the injected gas and its pressure, the dynamics of both the gas effect itself and of the reestablishment of higher levels of field emission when the gas is withdrawn, as well as several other observed phenomena which may also help to elucidate the mechanism responsible for the gas effect. After these various experimental observations are presented, they will then be discussed in terms of possible mechanisms.

\section{EXPERIMENT}

Figure 1 shows a schematic view of the apparatus employed for dark current measurement and the electrode geometry. The vacuum was obtained by using Pfeiffer HiPace $8067 \mathrm{~L} / \mathrm{s}$ turbo molecular secondary and DUO 5M primary pumps. Four gases were considered: $\mathrm{H}_{2}, \mathrm{He}, \mathrm{N}_{2}$, and Ar; these were injected using a Riber leakage valve. 


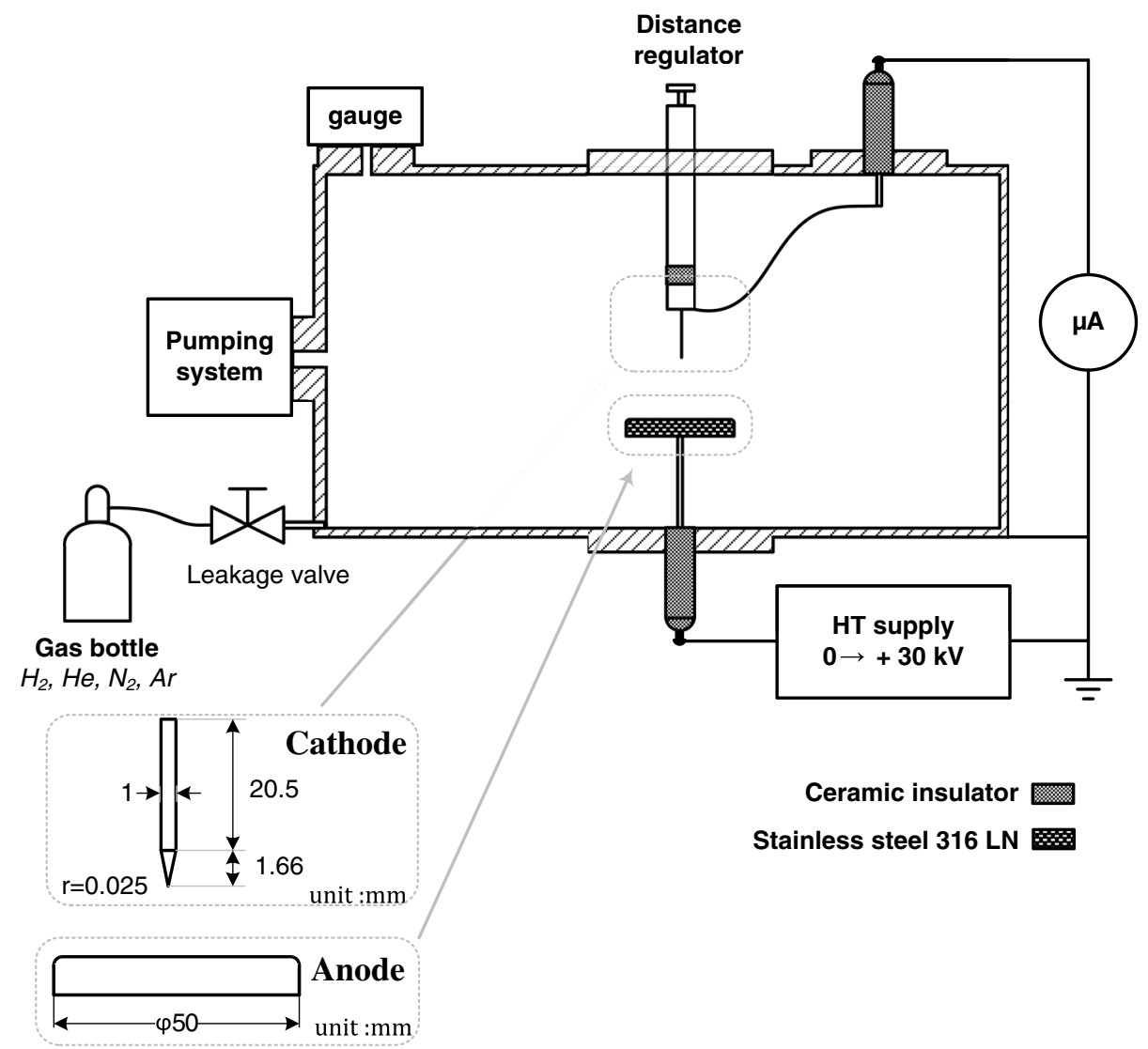

FIG. 1. Schematic view of the experimental apparatus.

Seeing that the pump was working constantly, the pressure was stabilized at the desired value when the flow of the injected and the pumped gas was equal. The vacuum pressure was measured using a compact PKR251 gauge (Pirani and cold cathode systems) with precision of $3 \times 10^{-7} \mathrm{~Pa}$, calibrated for $\mathrm{N}_{2}$. Corrective factors were used to obtain the correct pressure values for the other gases.

The gap spacing between electrodes was adjusted to $2 \mathrm{~cm}$ by using a micrometric distance regulator with a precision of $10 \mu \mathrm{m}$. Direct current positive voltage was applied to the planar electrode with a maximum value of $30 \mathrm{kV}$. The emission current was measured at the cathode by either a microammeter or by a high-impedance voltage measurement across a shunt resistor; measured intensities ranged from $0.1 \mathrm{nA}$ to $300 \mu \mathrm{A}$.

The typical experimental procedure consisted of measuring the emission current for periods of up to several hours at a pressure of $4 \times 10^{-6} \mathrm{~Pa}$ until it stabilized at which point gas was injected into the chamber. Other procedures were used depending on the goal of the particular experiment; these will be detailed in Sec. III.

The anode was disk shaped and made of $316 \mathrm{~L}$ stainless steel with $50 \mathrm{~mm}$ diameter and thickness of $8 \mathrm{~mm}$ with a rounded edge; the cathode was a cemented tungsten carbide rod with a $1 \mathrm{~mm}$ diameter and a pointed end with a $25 \mu \mathrm{m}$ radius of curvature as shown in Fig. 1. A macroscopic field enhancement factor of $\beta \cong 200$ was calculated for this geometry.

Figure 2 shows SEM micrographs of the tip of one of the tungsten carbide cathodes; image 2(a) is of a new tip, while

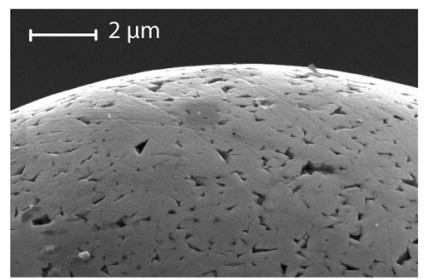

(a)

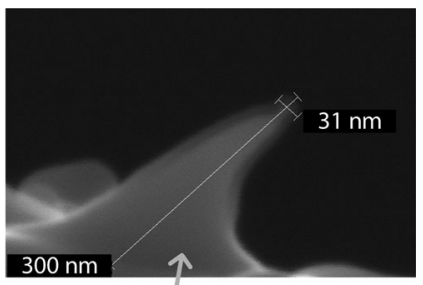

(b)

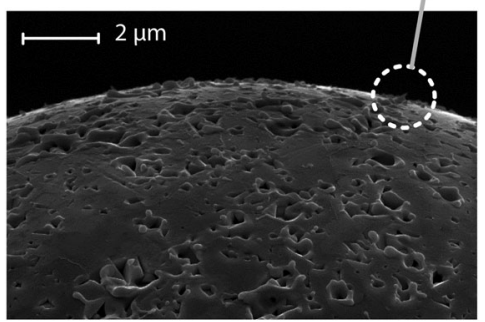

(c)

FIG. 2. SEM images of the tungsten carbide cathode (a) virgin cathode (b) submicron sized tip (c) cathode after breakdown. 
images 2(b) and 2(c) are of the same tip which has experienced several breakdowns (a discharge on the order of $>$ tens of amperes during a duration of $\sim 5 \mu \mathrm{s}$ ). All data to be presented will be concerned uniquely with emission from tips resembling those depicted in Figs. 2(b) and 2(c) because the current levels were higher, as well as more stable and repeatable. In the images, it can be observed that the breakdown led to the formation of nanometerscale features by partial melting of the surface. The field enhancement factor resulting from the combination of the macroscopic and microscopic morphologies was estimated to be as much as 2000 .

\section{RESULTS}

The results are to be presented as follows: an experiment with a pressure step change of about 3 orders is presented to demonstrate the dynamics of the gas effect on dark current and its recovery. Next, a series of experiments using four different gases with progressively increasing pressure illustrates the dependence of the gas effect on the gas nature and pressure. In the third section, the observation of a threshold of field/current level under which the gas effect is not observed is presented, and finally some aspects of the dynamics of the current recovery after gas exposure are investigated.

\section{A. Dynamics of the current decrease and recovery}

An example of the gas effect on field emission current is shown in Fig. 3. The current was stable at around $220 \mu \mathrm{A}$ for tens of minutes (not shown) at a pressure of $2 \times 10^{-5} \mathrm{~Pa}$ prior to the introduction of the gas. The applied voltage was reduced to zero prior to helium introduction, and then turned back on after the gas pressure had been adjusted to the desired value of $10^{-2} \mathrm{~Pa}$. At that point, the current decreased to a stable value of about $25 \%$ of its previous level. The current decrease is not immediate but has a time constant of $\sim 45 \mathrm{~s}$ and was fully stable after $5 \mathrm{~min}$ of gas

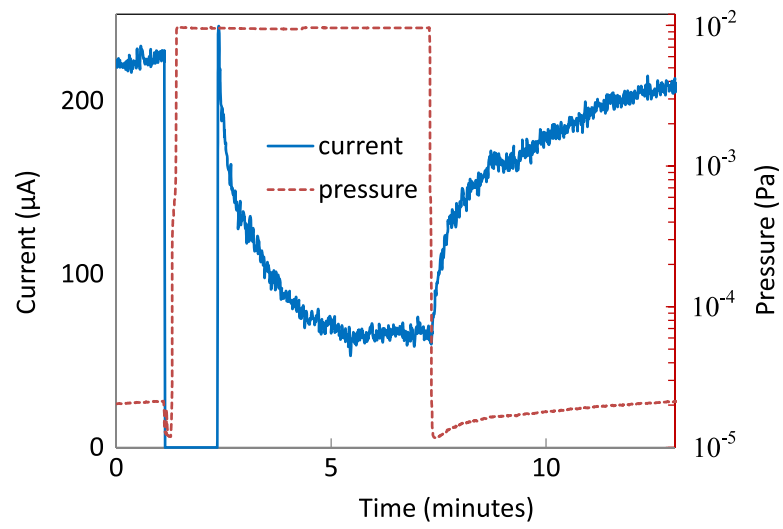

FIG. 3. Typical field emission current decrease and recovery caused by the injection and pumping down of helium: $2 \mathrm{~cm}$ gap distance, $30 \mathrm{kV}$ applied voltage. exposure, at which point the helium flow was cut off. The gas pressure takes only a few seconds to reach the lower value, after which the current began to recover. In this experiment, the recovery process has a similar dynamic to the current decrease process; after 5 minutes, it had recovered to nearly its initial value. Here it should be stated that, while the dynamic of the current decrease was reproducibly similar to that shown in Fig. 3 (i.e., exhibiting a time constant on the order of $<1$ minute), the current recovery dynamic is much more variable.

During the current recovery, the gas pressure is observed to increase - this phenomena of the gas pressure "following" the emission current is often observed in the present work and is likely due to desorption of adsorbed gas from surfaces which are heated by the emission current [13]. Much of the energy of the $30 \mathrm{keV}$ electrons arriving at the anode is dissipated as heat [7]; a separate experiment confirmed that at least $95 \%$ of the energy balance goes toward heating of the anode.

\section{B. Effect gas nature on current decrease}

The drop in field emission current was measured using four different gases: hydrogen, helium, argon, and nitrogen. The initial current value was variable (between 100 and $300 \mu \mathrm{A}$ ) and depended largely on the condition of the cathode: in general, the more breakdowns a cathode had been exposed to (after the initial one), the lower the current value at low pressure $\left(\sim 5 \times 10^{-6} \mathrm{~Pa}\right)$. For comparison between the different gases, only experiments with similar initial current values between $210 \mu \mathrm{A}$ and $260 \mu \mathrm{A}$ were selected to be presented here.

As illustrated in Fig. 4, when using the same electrode geometry and applied voltage, the gas pressure for each of the four different gases was increased progressively in 10 minute time steps. All four gases cause a reduction in current. However, there are clear differences between them, with nitrogen and argon having a stronger effect at lower

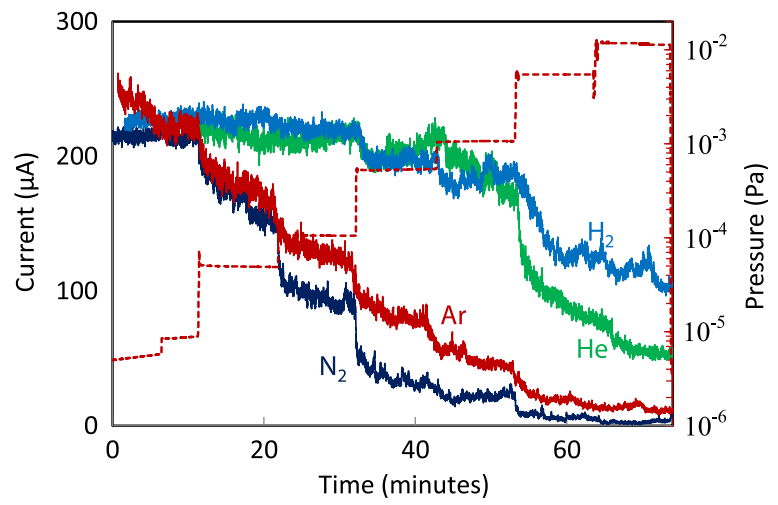

FIG. 4. Field emission current decrease for four different gases with increasing gas pressure: $2 \mathrm{~cm}$ gap distance, $30 \mathrm{kV}$ applied voltage. 


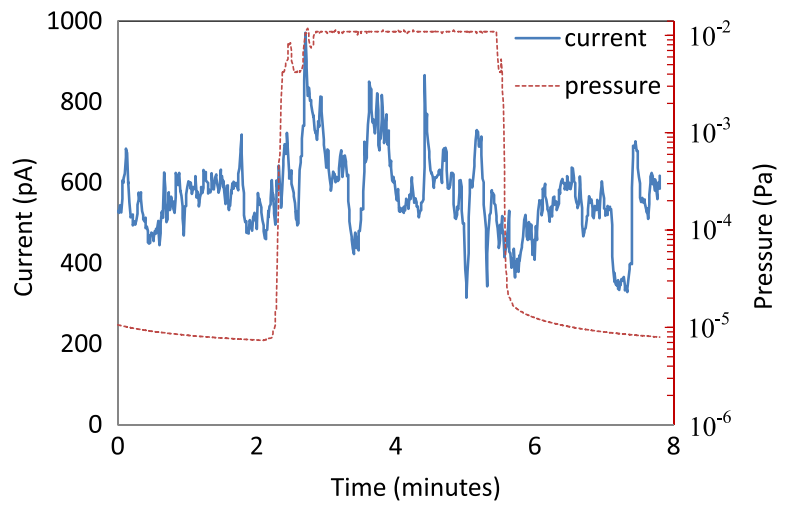

FIG. 5. Field emission current with nitrogen injection: $2 \mathrm{~cm}$ gap distance, $11 \mathrm{kV}$ applied voltage.

pressures than helium and hydrogen - this point will be discussed further in Sec. IV.

\section{Gas effect threshold}

A threshold in the field emission current (or the corresponding local electric field leading to said current), below which no gas effect took place was observed in the present work. For the same $2 \mathrm{~cm}$ gap distance but with $11 \mathrm{kV}$ of applied voltage, it is seen in Fig. 5 that the injection of nitrogen did not cause the current of $\sim 500 \mathrm{pA}$ to decrease.

Figure 6 demonstrates that for an intermediate applied voltage of $16 \mathrm{kV}$ and $\sim 1 \mu \mathrm{A}$ of initial current, the injection of nitrogen has a similar effect as for the cases presented in Figs. 3 and 4. Thus, the threshold for the observation of a current decrease caused by gas injection is somewhere in the nanoampere range (for a relatively small-scale system such as the one discussed here). Stabilization of the current between these ranges (i.e., in the nA range) was very difficult, a fact which is perhaps related to the existence of a gas effect threshold. Subsequent experiments showed that for the other gas types, there was also a threshold below which no gas effect was observed.

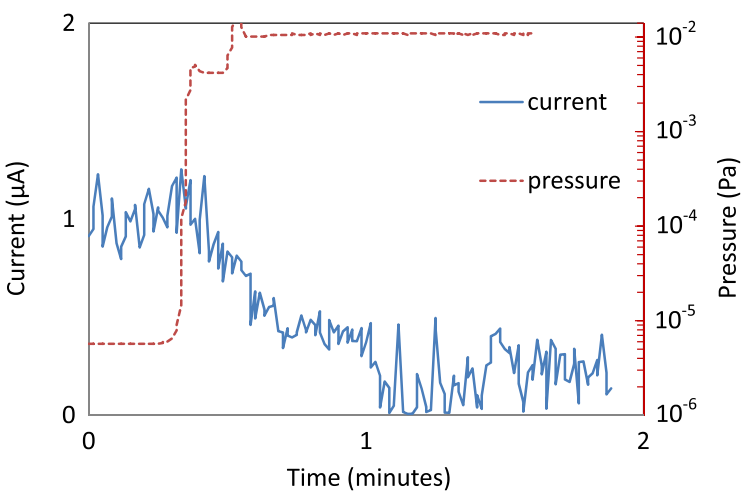

FIG. 6. Field emission current with nitrogen injection: $2 \mathrm{~cm}$ gap distance, $16 \mathrm{kV}$ applied voltage.

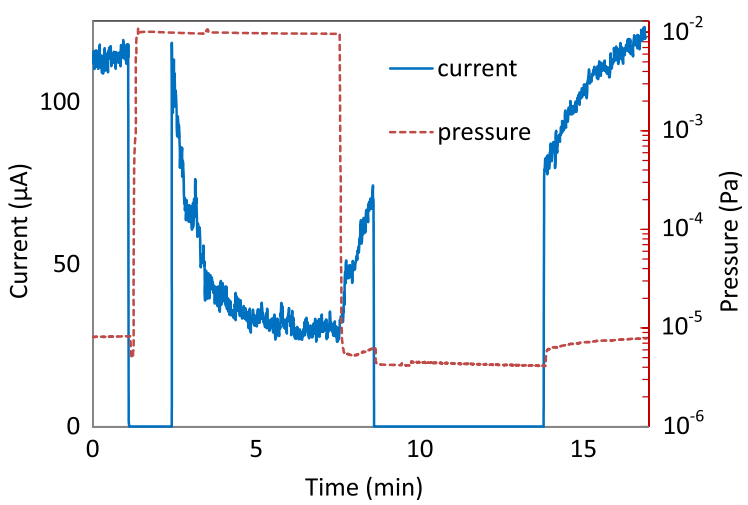

FIG. 7. Field emission current with helium injection followed by interrupted current recovery: $2 \mathrm{~cm}$ gap distance, $30 \mathrm{kV}$ applied voltage.

\section{Dynamics of the current recovery}

An experiment similar to the one depicted in Fig. 3 was performed to investigate the mechanism leading to the current recovery and is depicted in Fig. 7. After the gas injection was stopped and the current recovery had begun, the voltage supply was turned off for a period of 5 minutes. When the voltage was turned back on, the current recovery continued from about the same level that it had been at the moment of the voltage supply being turned off, and with similar dynamics, as if the process leading to the recovery had simply been interrupted. Another experiment (not shown) in which the electrodes were heated by a nearby filament to a temperature of $\sim 120^{\circ} \mathrm{C}$ during a longer duration interruption of the recovery showed a very similar result to the one shown in Fig. 7. Again it should be emphasized that the observed current recovery dynamic was not as repeatable as was the dynamic of the current drop. The current recoveries depicted in Figs. 3 and 7 had similar time constants to the current drops, making them easier to depict graphically; other current recoveries were often slower, and occasionally faster than the current drops.

A final experiment will be presented before passing to the discussion section, relating to the surface condition of the emitter prior to being exposed to gas at higher pressure. It was mentioned in Sec. II that the point-shaped cathodes were systematically exposed to breakdowns before they were used in experiments. Figure 8 shows a long duration experiment in which several breakdowns occurred. The experiment began with gas injection, followed by a relatively slow recovery during which a breakdown occurred, followed by several more breakdowns over a period of hours. The current decreases dramatically after each breakdown, and then increases slowly. The same type of current recovery after breakdown was always observed for new electrodes which had never been exposed to higher gas pressure in the presence of electric field. The time constants of the multiple current increases shown in Fig. 8 vary-some are similar to the current recoveries depicted in Figs. 3 and 7; others are slower. 


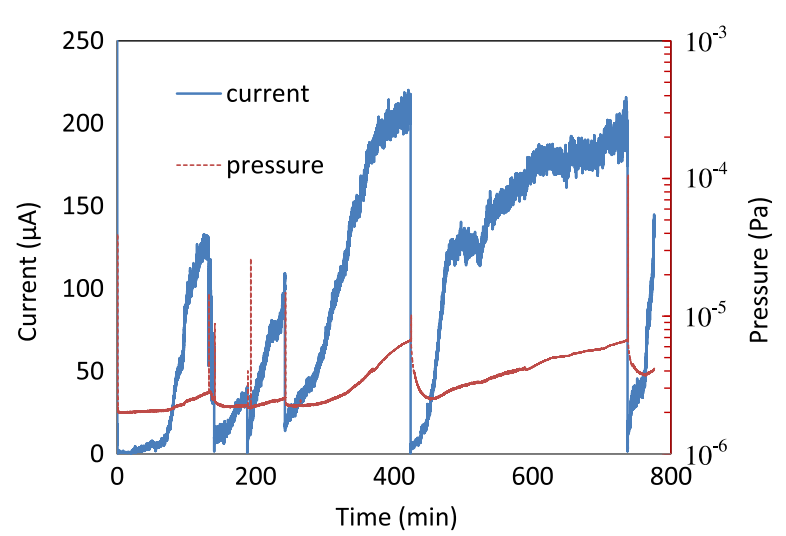

FIG. 8. Long-term experiment with multiple breakdowns followed by slow current increases. Each breakdown exhibits an immediate drop in current and a pressure spike.

\section{DISCUSSION}

In this section, it shall be endeavored to compare and contrast several different hypotheses for the mechanism of the gas effect in light of the data presented in Sec. III. First, each of the several hypotheses is presented with accompanying remarks, and then each of the major experimental observations will be discussed in separate sections.

It should be noted that, when materials other than tungsten carbide were used as cathodes (including pure tungsten and stainless steel), reduction of dark current and the current recovery were also observed. The points for these experiments were mechanically sharpened and had less reproducible results; however it can clearly be stated that the gas effect and current recovery observed here was not a special case involving tungsten carbide.

\section{A. Possible mechanisms}

\section{Field induced adsorption}

It is known that gas adsorption can be induced by strong electric fields [11,14]. Furthermore, it is known that adsorbed gases, by altering the work function for electron emission, may inhibit field emission [12,13]. Calculations show that the electric field necessary to create such an effect would have to be quite large, on the order of $2.0 \times 10^{10} \mathrm{~V} / \mathrm{m}[11,14]$.

The electric field along the axis between the point electrode and planar anode shown in Fig. 2 may be estimated as that between a parabola-shaped point and an infinite plane as given by Eq. (1).

$$
F(z)=\frac{\mathrm{V}}{\left(z+\frac{r}{2}\right) \ln \left(\frac{2 d+r}{r}\right)},
$$

where $\mathrm{V}$ is the applied voltage at the anode, $z$ is the axial position between the point cathode and the planar anode, $r$ is the radius of curvature of the point, and $d$ is the pointplane distance. This gives a field of $3.25 \times 10^{8} \mathrm{~V} / \mathrm{m}$ at the tip of the cathode for an applied voltage of $30 \mathrm{kV}$, interelectrode distance of $2 \mathrm{~cm}$, and tip radius of curvature of $25 \mu \mathrm{m}$. An additional field enhancement on the order of 60 would therefore be needed to attain the field strength theoretically needed for field-induced adsorption to take place. This sort of field enhancement is not out of the question considering the presence of microstructures such as the one seen in Fig. 2(b).

\section{Ionic bombardment}

Another mechanism for the reduction in field emission current by gas is the one originally proposed by Alpert [6] in which ions produced locally near microscopic field emitters bombard those emitters, leading to a reduction of their size or aspect ratio, thus reducing the current because of the reduced local electric field. This mechanism for field emission current reduction is arguably the most often seen in the literature, and is commonly referred to as gas conditioning or helium processing $[7,9,15]$.

A related mechanism, suggested by Latham [10], is that dielectric inclusions are responsible for the dark current emission, and ion bombardment of these inclusions leads to the reduction of the emission current. In this paper, since the zone at high field is very small, and images do not indicate the presence of any insulating material, it is difficult to speculate on the applicability of this mechanism to explain the present results.

\section{Changes in the surface coverage}

For the lower value of pressure in this paper on the order of $10^{-5} \mathrm{~Pa}$, according to a simple calculation using the Nernst-Knudsen equation, every surface site will be impacted by a gas atom/molecule in a time frame on the order of $1-10 \mathrm{~s}$. Therefore, (depending on the sticking coefficient for the particular gas) even at this "low" value of pressure, an equilibrium surface coverage should be established well before a particular experiment is performed. In much previous work $[13,16]$, much care was taken to have a gas-free cathode surface in order to study the effects of adsorption. These precautions have not been taken in this work; it should generally be assumed that the cathode surface coverage is at equilibrium. A mechanism may then be imagined as referred to in [13] in which ions created in the interelectrode gap "clean" by bombardment the surface of the cathode of adsorbed gas and thereby lead to the gas effect (this would of course require that the effect of adsorbed gas be to reduce the work function). Alternatively, instead of adsorbed gas, other contaminants could be involved such as organic material originating from the pump system lubricating oil [17-20].

\section{B. Gas nature}

The observations show clearly that the heavier gases argon and nitrogen have stronger inhibitory effects on field 


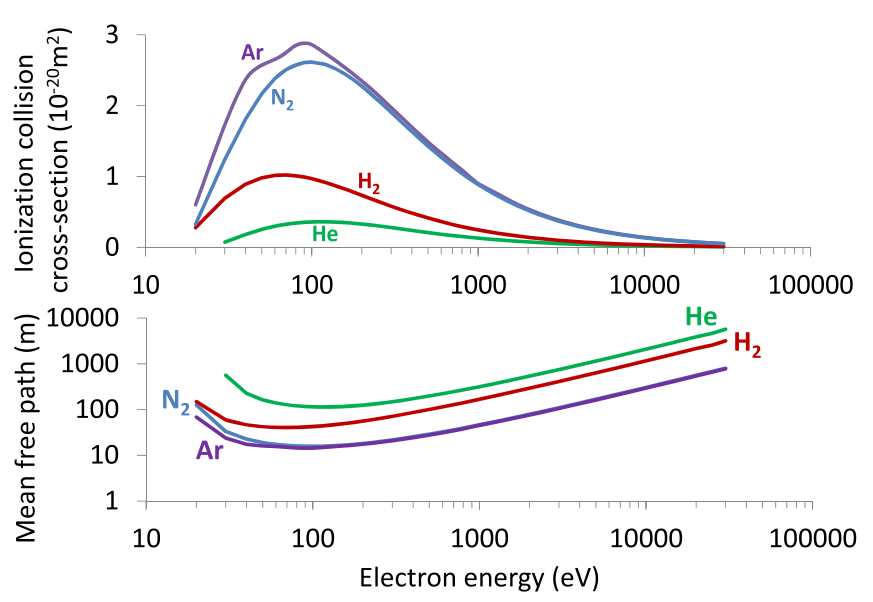

FIG. 9. Ionization collision cross sections and calculated mean-free path calculated at $10^{-2} \mathrm{~Pa}$ as functions of electron energy for $\mathrm{Ar}, \mathrm{N}_{2}, \mathrm{H}_{2}$, and $\mathrm{He}$.

emission than the lighter gases helium and hydrogen. There seems not to be a correlation between the effect with whether the gas is monatomic (noble, nonreactive) or diatomic (chemisorption, formation of covalent bonds with surface groups possible).

Figure 9 shows a comparison of the ionization cross sections and mean-free paths as a function of electron energy for $\mathrm{N}_{2}, \mathrm{H}_{2}, \mathrm{He}$ (data taken from NIST database), and Ar [21]. Comparison of Figs. 4 and 9 suggests that the dependence on gas nature of the magnitude of the gas effect could be related to ionization.

\section{Threshold for the gas effect}

The threshold for the observation of the gas effect is compatible with any of the hypotheses, by insufficient local electric field to induce adsorption for the field-induced adsorption mechanism, and by insufficient local electron density to induce bombardment for the others.

It can be mentioned here that occasionally when gas was injected during operation at current levels just above the threshold value (i.e., $\sim 1$ microampere), the current would decrease to a level well below the threshold (i.e., in the range of $\mathrm{pA}$ ). The current would then rest at this level for long durations ( $>20$ minutes) even if the gas pressure was then reduced (i.e., there was no current recovery in this case). The system being "forced below threshold" would imply that whatever mechanism which leads to the current recovery is inactivated.

\section{Current recovery}

The observations on the current recovery dynamic as seen in Figs. 3 and 7 pose greater challenges in their explanation than that of the current reduction. The adsorption mechanism would involve desorption of the additional adsorbed gas held in place by the large local electric field. A priori, this desorption should be immediate at the moment when the electric field is annulled, therefore, the interrupted recovery experiment shown in Fig. 7 should have led to an immediate recovery of the initial current when the field was turned back on. This result is therefore contradictory with the field-induced adsorption hypothesis for the gas effect.

The explanation for the current recovery in the context of the emitter erosion by ionic bombardment mechanism would necessarily involve the reconstruction of the field emitters during the recovery. Surface migration of tungsten was first suggested by Müller [22] after his invention of the field emission microscope, with further evidence of its existence and reversibility demonstrated shortly thereafter by Benjamin [16]. It should be noted that in both of these studies, much care had to be taken to start with a clean emitter, i.e., adsorbed gas free, because in the presence of gas, the emission images were seen to degrade with time due to adsorption (and/or bombardment). Again, in the present work, adsorbed gas should always be present (because of the minimum vacuum level of $\sim 10^{-5} \mathrm{~Pa}$ ) except perhaps in the seconds immediately following breakdown. Further work on migration of material in field emitters by Bettler [23] measured the activation energy for the transport of surface atoms in both directions (transport toward the emitter tip in the presence of field and toward the shank in the absence of field).

Other work may be found in the literature on similar phenomena to the current recovery, which may be referred to as a "deconditioning" [7]. Surface migration is also thought to be a contributor to breakdown $[24,25]$ and more recent work which has sought to model "asperity growth" has determined that Nottingham heating dominates over Joule heating [26]. Other interesting modeling work on the phenomenon makes quantitative predictions about emitter growth and proposes an explanation for the well-known "total voltage effect" $\left(\mathrm{V}_{\text {breakdown }} \sim \mathrm{d}^{0.6}\right)$ on breakdown based on emitter growth on flat planes [27]. In summary, the reconstruction of nanoscale emitters is a possible explanation for both the current recovery after the gas effect and the current increase observed after breakdowns in this work.

If adsorbed gas were responsible for a reduction in the work function, and therefore, the high initial value of current, and that ionic bombardment caused by the injection of the gas removed this adsorbed gas, leading to the current reduction, the current recovery would then be caused by the readsorption of gas after pumping down. This idea is also contradicted by the result shown in Fig. 7. It can also be mentioned that in an additional experiment (not shown) very similar to that in Fig. 7, but in which gas was injected (at $10^{-2} \mathrm{~Pa}$ ) during the current recovery interruption for several minutes (theoretically more than enough time to establish an adsorption-desorption equilibrium at this pressure) and then pumped down prior to reactivating the electric field, the same result was observed 
as that of Fig. 7, namely that the current after the interruption was equal to that before and continued to increase as if the process leading to the recovery had simply been interrupted. However, if a reduction of work function were due not to adsorbed gas but another substance such as organics from pump lubricating oil, the mechanism for the current recovery might not be simple adsorption from the gas phase, but surface diffusion from surfaces adjacent to the emission site. If this diffusion only takes place in the presence of the electric field, this mechanism would then be in agreement with the results of Fig. 7.

For the idea of ion implantation in a dielectric emitter, the current recovery would be caused by removal of the implanted ions by diffusion or some other mode of transport, which would be accelerated by local heating. In principle, the implanted ions should be trapped in the dielectric and their accumulation might even lead to physical damage to the dielectric structure as is known to occur in the case of helium implantation in tungsten, for example. Further speculation on this particular mechanism is beyond the scope of this paper.

The current recovery observed after a breakdown with an electrode which had never been exposed to gas at high pressure during field emission is however difficult to explain with the ion implantation, the field-induced adsorption, or the modification of gas adsorption equilibrium hypotheses. The other two hypotheses of ionic bombardment emitter erosion/metal migration and ionic bombardment surface cleaning/migration of contaminant such as lubricating oil are however consistent with the data in Fig. 8.

\section{E. Calculations for the ionic bombardment mechanism}

\section{Calculation of ion production}

Estimations were made of the production of ions for the four different cases and for three different zones along the axis of the gap between the point cathode and planar anode. The calculation was made based on the isopotentials calculated by integration of Eq. (1) and the corresponding mean-free path values seen in Fig. 9. The gap was discretized into small zones $(\Delta z)$ near the cathode, where the change in field strength is steep as a function of distance, and larger zones closer to the anode. The number of ions created per unit time (in equivalent amperes) was then calculated for each of these zones simply according to Eq. (2):

$$
I_{\text {ion }}=\frac{\Delta z}{(z)} I_{e^{-}}
$$

where $\Delta z$ is a portion of the gap distance with an average mean-free path value of $\lambda(z)$; the ion production in that portion is then simply the ratio of the distance traveled by an electron to its mean-free path over that zone, multiplied by the electronic current. Table I summarizes the results of
TABLE I. Summary of ionic current estimations.

\begin{tabular}{lccc}
\hline \hline & Zone "4 $\mu \mathrm{m}$ " & Zone "close" & Zone "far" \\
\hline $\mathrm{e}^{-}$energy & $20-10^{3} \mathrm{eV}$ & $10^{3}-10^{4} \mathrm{eV}$ & $10^{4}-3 \times 10^{4} \mathrm{eV}$ \\
Distance from & $\sim 0-4 \mu \mathrm{m}$ & $\sim 4-140 \mu \mathrm{m}$ & $140 \mu \mathrm{m}-2 \mathrm{~cm}$ \\
cathode & & & \\
Ion current:nitrogen & $1.2 \times 10^{-11} \mathrm{~A}$ & $6.8 \times 10^{-11} \mathrm{~A}$ & $2.9 \times 10^{-9} \mathrm{~A}$ \\
Hydrogen & $3.9 \times 10^{-12} \mathrm{~A}$ & $1.8 \times 10^{-11} \mathrm{~A}$ & $7.6 \times 10^{-10} \mathrm{~A}$ \\
Helium & $1.8 \times 10^{-12} \mathrm{~A}$ & $9.9 \times 10^{-12} \mathrm{~A}$ & $4.2 \times 10^{-10} \mathrm{~A}$ \\
Argon & $1.3 \times 10^{-11} \mathrm{~A}$ & $7.0 \times 10^{-11} \mathrm{~A}$ & $3.0 \times 10^{-9} \mathrm{~A}$ \\
\hline \hline
\end{tabular}

this calculation, made using $I_{e}=100 \mu \mathrm{A}$ and a pressure of $10^{-2} \mathrm{~Pa}$ :

The ion current is dominated by ions created "far" (defined here somewhat arbitrarily as $z>140 \mu \mathrm{m}$ from the cathode tip), despite the lower value of collision cross section for the electrons transiting that zone. However, there is a non-negligible amount produced within the "close" $(4 \mu \mathrm{m}<z<140 \mu \mathrm{m})$ and "very close" $(z<4 \mu \mathrm{m})$ regions as well. Each of these zones consists of many $\Delta z$ discretizations.

\section{Erosion of emitters}

Estimations about the ion flux impacting the cathode tip may now be made. Considering the tip of the cathode delimited by its radius of curvature $(r=25 \mu \mathrm{m})$, a surface area of about $4 \times 10^{-9} \mathrm{~m}^{2}$ is found. Taking an interatomic distance for tungsten carbide as $0.28 \mathrm{~nm}$, a surface site would be associated with an area on the order of $8 \times 10^{-20} \mathrm{~m}^{2}$, giving a number of surface sites on the cathode tip equal to $4 \times 10^{-9} / 8 \times 10^{-20}=5 \times 10^{10}$ sites. The time then, for every surface site to be impacted by an ion, considering the sum of the ion production in all three zones for nitrogen gives a time constant of $5 \times 10^{10} / 1.9 \times 10^{10} \mathrm{~s}^{-1}=2.6 \mathrm{~s}$.

This calculation can be thought of as the time needed to deposit a single monolayer on the surface of the entire cathode tip, assuming all ions produced in the far region of the gap impact in that zone, which is perhaps an overestimate. For the deposition of a single monolayer to lead to the gas effect would necessarily imply that an emitter be extinguished by a single impact event, an hypothesis which might accord with an implantation or adsorption mechanism, but which would not suffice in the case of a mechanical erosion of nanostructure mechanism.

For the mechanical erosion of a field emitter to be responsible for the reduction in field emission, it would need to erode the field emitter in such a way as to reduce its field enhancement value roughly as shown in Fig. 10. For a structure such as the one seen in Fig. 2(b), this would correspond at a first approximation to a $10 \%$ reduction in its aspect ratio. Consider then such a structure: its tip is very small compared to the macroscopic one considered earlier, at $\sim 7 \times 10^{-16} \mathrm{~m}^{2}$, giving a total number of sites of $\sim 10^{4}$. If we consider only the very close ion flux from Table I, this would mean that every site will be impacted by an ion in 


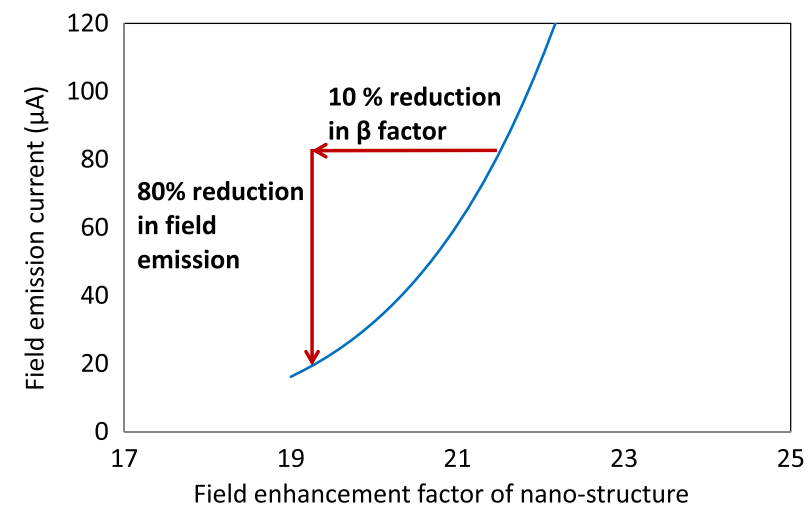

FIG. 10. Calculation of the field emission using the FowlerNordheim equation from a cathode at $300 \mathrm{MV} / \mathrm{m}$ with an additional field enhancement.

about $\sim 130 \mu \mathrm{s}$. To account for the gas effect, as shown in Fig. 10, the aspect ratio of this emitter would need to be reduced by about $10 \%$, meaning that about $30 \mathrm{~nm} / 0.28 \mathrm{~nm}=107$ layers would need to be removed. If there is perfect sputtering efficiency (and no ions miss their targets), this could happen in about $14 \mathrm{~ms}$, so a sputtering efficiency of $\sim 3 \times 10^{-4}$ would lead to a time constant of $\sim 45 \mathrm{~s}$. The sputtering efficiency of tungsten by argon ions falls in the region of $\sim 10^{-3}-10^{-1}$ for energies on the order of 20-1000 eV [28]. Tungsten carbide being harder than pure tungsten, these values might reasonably be expected to be lower for the present calculation. Another consideration is that, while ions created far from the emission site will then impact the cathode with higher energy, they will also be less likely to impact the emitter. In addition since in experiment the current is dropping during the bombardment, the constant current of $100 \mu \mathrm{A}$ in this simple estimation gives by definition an overestimate of the speed of erosion.

Attempts were made to take images of the cathode surface shown in Fig. 2 after it had been subjected to a gas effect. No obvious morphological changes were observed, however, the fact that only a change on the order of $10 \%$ aspect ratio might be expected, combined with the difficulty of locating nanoscale structures on a surface as depicted in Fig. 2, and viewing the same ones at the same angles as in the before-gas effect images, could mean that the changes could easily be unobservable or just simply missed.

\section{CONCLUSION}

The reduction of field emission current from a tungsten carbide emitter caused by the presence of gas has been investigated. The injection of four different gases at pressures of $\sim 10^{-3}-10^{-2} \mathrm{~Pa}$ resulted in a reduction in the field emission, with larger effects at lower pressure seen for the heavier gases, nitrogen and argon, than the lighter gases, helium and hydrogen. A threshold in the value of electric field/emission current level was observed, under which no reduction in current is observed to be caused by the gas. When the gas is removed, a mechanism leading to the reestablishment of the emission takes place. If this current "recovery" is interrupted by canceling the field, the recovery process stops and then restarts when the field is reapplied. Several different mechanisms were presented and compared to explain these results. Its seems very likely from the results that the decrease in current is due to ionic bombardment of the cathode, likely by erosion of nanoscale emitters or perhaps by the removal of contaminants which change the work function. The corresponding mechanism leading to the current recovery is proposed to be due to the migration of metal under the influence of the high electric field to reform the eroded emitters, or alternatively to be due to surface diffusion of contaminants under the influence of the field.

[1] R. H. Fowler and L. Nordheim, Proc. R. Soc. A 119, 173 (1928).

[2] A. Simonin, H. de Esch, L. Doceul, L. Christin, F. Faisse, and F. Villecroze, Fusion Eng. Des. 88, 1 (2013).

[3] R. S. Hemsworth, A. Tanga, and V. Antoni, Rev. Sci. Instrum. 79, 02C109 (2008).

[4] R. Hemsworth, H. Decamps, J. Graceffa, B. Schunke, M. Tanaka, M. Dremel, A. Tanga, H. P. L. D. Esch, F. Geli, J. Milnes, T. Inoue, D. Marcuzzi, P. Sonato, and P. Zaccaria, Nucl. Fusion 49, 045006 (2009).

[5] R. V. Latham, High Voltage Vacuum Insulation: Basic Concepts and Technological Practice (Elsevier, New York, 1995).

[6] D. Alpert, D. Lee, E. M. Lyman, and H. E. Tomaschke, J. Appl. Phys. 38, 880 (1967).

[7] G. P. Beukema, Physica (Amsterdam) 61, 259 (1972).

[8] R. Bloomer and B. Cox, Vacuum 18, 379 (1968).

[9] B. Bonin, Vacuum 46, 907 (1995).

[10] S. Bajic, A. M. Abbot, and R. V. Latham, IEEE Trans. Electron. Insul. 24, 891 (1989).

[11] R. L. C. Wang, H. J. Kreuzer, and R. G. Forbes, Surf. Sci. 350, 183 (1996).

[12] R. Gomer and J. K. Hulm, J. Chem. Phys. 27, 1363 (1957).

[13] A. Zeitoun-Fakiris and B. Juttner, J. Phys. D 21, 960 (1988).

[14] N. Ernst, W. Drachsel, Y. Li, J. H. Block, and H. J. Kreuzer, Phys. Rev. Lett. 57, 2686 (1986).

[15] J. J. Maley, J. Vac. Sci. Technol. 8, 697 (1971).

[16] M. Benjamin and R. O. Jenkins, Proc. R. Soc. Math. Phys. Sci. 176, 262 (1940).

[17] M. T. Postek, Scanning 18, 269 (1996).

[18] E. I. Rau, Appl. Surf. Sci. 254, 2110 (2008).

[19] M. Amman, J. W. Sleight, D. R. Lombardi, R. E. Welser, M. R. Deshpande, M. A. Reed, and L. J. Guido, J. Vac. Sci. Technol. B 14, 54 (1996).

[20] L. Holland, Vacuum 20, 175 (1970).

[21] R. Rejoub, B. G. Lindsay, and R. F. Stebbings, Phys. Rev. A 65, 042713 (2002).

[22] E. W. Müller, Z. Phys. 106, 541 (1937). 
[23] P. C. Bettler and F. M. Charbonnier, Phys. Rev. 119, 85 (1960).

[24] G. N. Fursey, IEEE Trans. Electron. Insul. 20, 659 (1985).

[25] C.Z. Antoine, F. Peauger, and F. Le Pimpec, Nucl. Instrum. Methods Phys. Res. Sect. Accel. Spectrometers Detect. Assoc. Equip. 670, 79 (2012).
[26] K. L. Jensen, Y. Y. Lau, D. W. Feldman, and P. G. O'Shea, Phys. Rev. ST Accel. Beams 11, 081001 (2008).

[27] G. L. Bilbro, J. Vac. Sci. Technol. B 20, 757 (2002).

[28] R. Behrisch, Sputtering by Particle Bombardment (Springer-Verlag, Berlin, 1981). 